\title{
Management of metastatic urothelial cancer: the role of surgery as an adjunct to chemotherapy
}

\author{
Robert S. Svatek, MD; ${ }^{*}$ Arlene Siefker-Radtke, MD; ${ }^{\dagger}$ Colin P. Dinney, MD*
}

\begin{abstract}
Metastatic or unresectable disease is identified in approximately $20 \%$ of patients presenting with invasive urothelial cancer. In addition, up to $50 \%$ of patients will develop metastases following radical cystectomy for clinically localized disease. Multiagent cisplatin-based chemotherapy is considered standard first-line treatment for these patients. Although urothelial cancer is considered a chemosensitive tumour, metastatic disease is associated with poor prognosis and short-term survival. Here, we review the role of a multidisciplinary approach to treating patients with metastatic urothelial cancer.
\end{abstract}

Can Urol Assoc J 2009;3(Supp|4):S228-31

M etastatic or unresectable disease is identified in approximately $20 \%$ of patients presenting with invasive urothelial cancer. In addition, up to $50 \%$ of patients will develop metastases following radical cystectomy for clinically localized disease. Multiagent cisplatinbased chemotherapy is considered standard first-line treatment for these patients. Although urothelial cancer is considered a chemosensitive tumour, metastatic disease is associated with poor prognosis and short-term survival. In this paper, we review the role of a multidisciplinary approach to treating patients with metastatic urothelial cancer.

\section{Chemotherapy}

Developed in the 1980s, the combination of methotrexate, vinblastine, doxorubicin, and cisplatin (MVAC) is now one of the most commonly used regimens for metastatic urothelial cancer. ${ }^{1}$ Prospective trials have demonstrated the superiority of MVAC over single-agent chemotherapy. ${ }^{2,3}$ In their initial report, Sternberg and colleagues reported $72 \%$ overall response and $36 \%$ complete response rates among patients with metastatic urothelial cancer. Loehrer and colleagues demonstrated response rates of 39\% in MVAC-treated patients compared with $12 \%$ in those treated with single-agent cisplatin. ${ }^{3}$ In addition, overall survival (OS) was significantly improved for the combination treatment group (12.5 vs. 8.2 months).
Response to cisplatin-based chemotherapy can be improved with the use of increased dose-intensity regimens. High-dose-intensity MVAC (HD-MVAC) has been shown to be superior to standard MVAC with regard to complete remission rate, progression-free survival and 2-year survival. ${ }^{4} \mathrm{HD}-\mathrm{MVAC}$ given with granulocyte colony-stimulating factor (G-CSF) allows for the delivery of twice the dose of cisplatin and doxorubicin with less toxicity and fewer dose delays. The benefit of this approach is supported by a phase 3 trial which observed a 2-year progression-free survival of $24.7 \%$ in the HD-MVAC plus G-CSF arm compared to $11.6 \%$ in patients treated with standard MVAC. ${ }^{4}$

In an effort to avoid treatment-related toxicity associated with MVAC, other regimens have been evaluated for patients with metastatic urothelial cancer. MVAC can be associated with severe complications such as neutropenia, infectious complications, mucositis, gastrointestinal and renal toxicity. These side effects are especially problematic in the urothelial cancer population, which has a large proportion of older patients with significant comorbidities.

Gemcitabine is a nucleoside analogue that has demonstrated activity and safety in patients as a single agent in phase 2 trials. ${ }^{5} \mathrm{~A}$ large phase 3 trial compared gemcitabine plus cisplatin (GC) to standard MVAC and reported similar OS, time to progressive disease and time to treatment failure. ${ }^{6}$ More grade 3 to 4 anemia and thrombocytopenia were observed in the GC arm, but the rates of grade 3 to 4 neutropenia, mucositis and incidence of neutropenic fever were greater in the MVAC arm. Although GC demonstrated similar activity compared to MVAC, the trial was powered to detect a $33 \%$ difference between the groups and not powered as an equivalence study. Moreover, GC has not been formally compared to HD-MVAC. Nevertheless, these study results showing comparable survival rates and a somewhat improved toxicity profile have led to the adoption of GC as front-line therapy at many centres.

Cisplatin-based systemic chemotherapy has been the mainstay of primary treatment for patients with urothelial carcinoma presenting with metastasis or unresectable disease. However, with the realization that, despite high response rates achieved with chemotherapy, few patients experience long-term survival, many centres have adopted 
a multidisciplinary approach with surgical consolidation of initially unresectable or metastatic disease. Most frequently, this occurs in patients with tumours initially adherent to the pelvic sidewall or with regional node involvement, but surgical consolidation has also been reported in nonregional nodal involvement and in visceral metastases.

There is a limited amount of data and no guidelines exist on the appropriate application and timing of surgery for patients with metastatic disease. Most centres would consider surgical consolidation with curative intent only for those patients exhibiting a response to systemic chemotherapy. Patients who do not respond to primary chemotherapy have a guarded prognosis and are unlikely to benefit from surgical excision of the primary tumour or metastatic disease. Any additional therapy should be considered as palliative. In selected patients, palliative radical cystectomy can be considered to relieve symptoms attributable to local tumour burden, such as pain, recurrent bleeding or fistula formation. ${ }^{7}$ In addition, palliative radiotherapy may provide rapid relief of tumour-related symptoms, especially hematuria. ${ }^{8} \mathrm{~A}$ large multi-centre trial demonstrated symptomatic improvement, such as hematuria, urinary frequency, dysuria or nocturia, in $68 \%$ of patients deemed unsuitable for curative treatment. ${ }^{9}$ The usefulness of palliative radiotherapy, however, must be considered within the context of the transitory symptomatic improvement and the poor survival for these patients.

\section{Surgical consolidation}

\section{Rationale}

The rationale for postchemotherapy surgery assumes that patients with complete responses to chemotherapy will still experience a high rate of disease relapse at the responding site(s) of disease. Dimopoulos and colleagues reviewed the outcomes for 58 consecutive patients with metastatic disease who relapsed after prior response to cisplatin-based chemotherapy. ${ }^{10}$ Of the patients presenting initially with local-regional metastasis, $74 \%$ had relapse at the site of response and $26 \%$ had visceral metastases at relapse. This pattern of relapse supports the rationale for consolidation therapy with surgery after maximum response to chemotherapy. The outcomes for patients undergoing surgical consolidation are dependent on the location of the disease (regional nodal, nonregional nodal, and visceral metastasis) as discussed below.

\section{Radical cystectomy and regional nodal dissection}

Several groups have reported outcomes for patients with metastatic or unresectable disease treated with postchemotherapy radical cystectomy and regional nodal dissection. ${ }^{11,12}$ Dodd and colleagues reported outcomes for 4 patients with unresectable disease who all achieved a complete response to MVAC. Residual disease was found in $75 \%$ of patients and $2(50 \%)$ patients were alive at 5 years. In addition, they reported outcomes for 15 patients with regional nodal involvement who underwent MVAC followed by surgery. Of these patients, $6(40 \%)$ were alive at 5 years. In their series, patients with unresectable disease and those with regional nodal involvement did far better than those with visceral metastasis. Similarly, Miller and colleagues noted that patients with liver, lung or bone involvement had significantly decreased survival compared to patients without visceral metastasis. ${ }^{12}$ A phase 3 trial comparing MVAC to fluorouracil, interferon alfa-2b and cisplatin (FAP) included 20 patients initially presenting with unresectable disease or nodes below the renal hila who were treated with chemotherapy followed by surgical consolidation. The median survival for this group of patients was 55.2 months from resection and 11 (55\%) were free of disease with a median follow-up of 51.8 months. A similar group of patients undergoing surgical consolidation at the Memorial Sloan-Kettering Cancer Center identified 9 of 20 (45\%) patients alive at 5 years. ${ }^{11}$

It is recommended that patients presenting with clinically positive nodes diagnosed by abdominal imaging be treated with multiagent chemotherapy. ${ }^{13}$ Our current approach to selecting patients for subsequent surgical consolidation is determined by the response to chemotherapy and the remaining tumour burden. Patients with a complete response are offered surgical consolidation while alternative chemotherapy is recommended for minimal or nonresponders. In patients with a partial response, we assess the residual tumour burden with nodal biopsy and cystoscopy. Generally, patients with negative nodal biopsies and limited tumour burden on cystoscopy are offered surgical consolidation.

Gross nodal involvement identified at the time of surgery does not necessitate abandonment of radical cystectomy or nodal dissection. Survival for these patients has been reported by Herr and colleagues. ${ }^{14}$ In that series, longterm survival was observed in $24 \%$ of 84 patients with grossly positive nodal disease at cystectomy and the median survival time was 19 months for all patients. ${ }^{14}$ As pointed out in the editorial published subsequent to that study, the benefit of radical cystectomy with nodal dissection was confounded by the presence of adjuvant chemotherapy which was given to an undisclosed number of patients. ${ }^{15}$ At our institution, patients with grossly positive nodal disease discovered at the time of surgery are offered additional adjuvant multiagent chemotherapy. In addition, the presence of gross nodal involvement at the time of cystectomy does not oblige the surgeon to perform an ileal-conduit diversion as continent orthotopic diversions have been safely utilized in this setting. ${ }^{16}$ 
In summary, patients undergoing surgical consolidation for unresectable disease and/or regional nodal metastasis are at considerable risk for disease recurrence, but survival rates of $45 \%$ to $55 \%$ have been reported for selected patients. These rates are considerably improved over the $13 \%$ to $30 \%$ survival rates for patients undergoing surgical consolidation for visceral metastasis (see visceral metastasectomy below).

\section{Non-regional nodal dissection}

The benefit of postchemotherapy retroperitoneal lymph node dissection (RPLND) for patients with nonregional nodal metastasis has been examined in a prospective fashion at the MD Anderson Cancer Center (MDACC). ${ }^{17}$ Sweeney and colleagues reported on the resection of clinically detectable retroperitoneal nodes in 11 patients after response to chemotherapy. Seven patients eventually developed recurrence outside of the original surgical field while 4 (36.4\%) patients were rendered free of disease as a result of surgical consolidation. In addition, they observed that patients with viable tumour in less than 2 lymph nodes had improved survival compared to those with viable tumour in more than 2 lymph nodes. Thus RPLND has curative potential in select patients with nonregional nodal metastasis.

\section{Visceral metastasectomy}

The role of metastasectomy in patients with advanced urothelial cancer is supported by several small retrospective series. Cowles and colleagues first reported surgery for metastatic urothelial cancer. ${ }^{18}$ They observed long-term disease control in 6 patients after the resection of a solitary pulmonary metastasis. A subsequent study from MDACC addressed the role of metastasectomy. Siefker-Radtke and colleagues reported outcomes for 31 patients undergoing postchemotherapy resection of metastatic lung $(77 \%)$, brain $(7 \%)$, skin $(3 \%)$ and distant lymph nodes $(13 \%) .{ }^{19}$ Median OS from the time of surgery was 23 months and a 33\% 5-year survival rate was observed.

Recently published data from contemporary cohorts lend further support to the use of metastasectomy in urothelial cancer. Abe and colleagues observed a median survival of 42 months in 12 patients who underwent metastasectomy at multiple sites including the lung, cervical lymph nodes and extended abdominal lymph nodes. ${ }^{20}$ Pathology of metastatic lesions revealed viable cancer in $83 \%$ of cases. Lehmann and colleagues reported outcomes for 44 patients from 15 different German centres, with distant metastases of the urothelial or upper urinary tract who underwent complete resection of all detectable metastases. ${ }^{21}$ OS from the time of resection was 27 months and 7 patients (15.9\%) survived more than 2 years without disease progression.
Recently, Siefker-Radtke and colleagues presented data from a phase II randomized, 4-regimen selection trial of sequential chemotherapy for patients with metastatic or unresectable urothelial cancer. In that report, 35 patients underwent surgical consolidation, including 24 with nodal metastasis, 6 with tumour fixed to the pelvic sidewall and 5 with other metastasis such as lung, brain, abdominal wall or ileum. Five-year OS for these patients was $29 \%$, and patients undergoing surgical consolidation after a $90 \%$ or greater response to chemotherapy showed the most improvement (40\% 5-year OS vs. $10 \% 5$-year OS if less than $90 \%$ response to chemotherapy).

The available evidence suggests that a select group of patients benefit from surgical consolidation of visceral metastases. This approach has been used most frequently in the setting of lung metastases, and more rarely in other organs, such as liver. While there are no standard guidelines in this setting, our current approach is to consider surgical consolidation of visceral metastases for those patients with tumour at 1 distant site who have responded well to chemotherapy and have no evidence of rapid progression elsewhere. In addition, we routinely observe patients for 3 to 6 months following chemotherapy to exclude rapid progression before considering surgical consolidation.

\section{Conclusion}

Long-term survival is infrequent but possible for some patients with metastatic urothelial cancer. MVAC has been considered the standard regimen since its introduction in the 1980s, but considerable efforts have been made to improve the efficacy and minimize the toxicity of this regimen. Retrospective studies have supported a role for surgical consolidation with curative intent for patients exhibiting response to primary chemotherapy. At the present time, surgery is generally considered appropriate for consolidation of regional lymph nodes, but is deemed investigational for more distant nodes and visceral metastases. A considerable number of these patients will experience disease progression and more accurate measures of disease response to chemotherapy, such as biologic markers or improved cross-sectional imaging, are needed to improve selection of candidates for surgical consolidation.

From *The University of Texas MD Anderson Cancer Center, Department of Urologic Oncology; TThe University of Texas MD Anderson Cancer Center, Department of Genitourinary Medical Oncology, Houston, TX

Competing interests: None declared.

This paper has been peer-reviewed. 


\section{References}

1. Sternberg CN, Yagoda A, Scher HI, et al. Preliminary results of M-VAC (methotrexate, vinblastine, doxorubicin and cisplatin) for transitional cell carcinoma of the urothelium. J Urol 1985;133:403-7.

2. Sternberg CN, Yagoda A, Scher HI, et al. M-VAC (methotrexate, vinblastine, doxorubicin and cisplatin) for advanced transitional cell carcinoma of the urothelium. J Urol 1988;139:461-9.

3. Loehrer PJ, Sr., Einhorn LH, Elson PJ, et al. A randomized comparison of cisplatin alone or in combination with methotrexate, vinblastine, and doxorubicin in patients with metastatic urothelial carcinoma: a cooperative group study. I Clin Oncol 1992;10:1066-73.

4. Sternberg CN, de Mulder PH, Schornagel JH, et al. Randomized phase III trial of high-dose-intensity methotrexate, vinblastine, doxorubicin, and cisplatin (MVAC) chemotherapy and recombinant human granulocyte colony-stimulating factor versus classic MVAC in advanced urothelial tract tumors: European Organization for Research and Treatment of Cancer Protocol no. 30924. J Clin Oncol 2001;19: 2638-46.

5. Hussain SA, James ND. The systemic treatment of advanced and metastatic bladder cancer. Lancet Oncol 2003;4:489-97.

6. von der Maase H, Hansen SW, Roberts JT et al. Gemcitabine and cisplatin versus methotrexate, vinblastine, doxorubicin, and cisplatin in advanced or metastatic bladder cancer: results of a large, randomized, multinational, multicenter, phase III study. J Clin Oncol 2000;18:3068-77.

7. Stenzl A, Cowan NC, De Santis M, et al. The Updated EAU Guidelines on Muscle-Invasive and Metastatic Bladder Cancer. Eur Urol 2009:55:815-25.

8. Errazquin Saenz de Tejada L, Gonzalez Ferreira JA, Mesa Saenz CM, et al. [Palliative radiotherapy of bladder cancer]. Arch Esp Urol 1999;52:627-36.

9. Duchesne GM, Bolger JJ, Griffiths $G 0$, et al. A randomized trial of hypofractionated schedules of palliative radiotherapy in the management of bladder carcinoma: results of medical research council trial BA09. Int J Radiat Oncol Biol Phys 2000;47:379-88.

10. Dimopoulos MA, Finn L, Logothetis CJ. Pattern of failure and survival of patients with metastatic urothelial tumors relapsing after cis-platinum-based chemotherapy. J Urol 1994;151:598-600; discussion 600-1.
11. Dodd PM, McCaffrey JA, Herr $\mathrm{H}$ et al. Outcome of postchemotherapy surgery after treatment with methotrexate, vinblastine, doxorubicin, and cisplatin in patients with unresectable or metastatic transitional cell carcinoma. J Clin Oncol 1999;17:2546-52.

12. Miller RS, Freiha FS, Reese JH, et al. Cisplatin, methotrexate and vinblastine plus surgical restaging for patients with advanced transitional cell carcinoma of the urothelium. J Urol 1993;150:65-9.

13. Evans CP, Swanson DA. What to do if the lymph nodes are positive. Semin Urol Oncol 1996;14:96-102.

14. Herr HW, Donat SM. Outcome of patients with grossly node positive bladder cancer after pelvic lymph node dissection and radical cystectomy. I Urol 2001;165:62-4; discussion 64.

15. Thompson IM. Re: Outcome of patients with grossly node positive bladder cancer after pelvic lymph node dissection and radical cystectomy. J Urol 2001;166:630.

16. Lebret $T$, Herve JM, Yonneau L, et al. After cystectomy, is it justified to perform a bladder replacement for patients with lymph node positive bladder cancer? Eur Urol 2002;42:344-9; discussion 349.

17. Sweeney $P$, Millikan $R$, Donat $M$, et al. Is there a therapeutic role for post-chemotherapy retroperitoneal lymph node dissection in metastatic transitional cell carcinoma of the bladder? J Urol 2003;169:2113-7.

18. Cowles RS, Johnson DE, McMurtrey MJ. Long-term results following thoracotomy for metastatic bladder cancer. Urology 1982;20:390-2.

19. Siefker-Radtke AO, Walsh GL, Pisters $L L$, et al. Is there a role for surgery in the management of metastatic urothelial cancer? The M. D. Anderson experience. J Urol 2004;171:145-8.

20. Abe T, Shinohara N, Harabayashi T, et al. Impact of multimodal treatment on survival in patients with metastatic urothelial cancer. Eur Urol 2007:52:1106-13.

21. Lehmann J, Suttmann H, Albers $P$, et al. Surgery for Metastatic Urothelial Carcinoma with Curative Intent: The German Experience (AUO AB 30/05). Eur Urol 2009;55:1293-9.

Correspondence: Dr. Colin P. Dinney, Professor, Departmental Chair, Division of Urology, MD Anderson Cancer Center, 1515 Holcombe Blvd, Houston, TX 77030; cdinney@mdanderson.org 